\title{
In-Situ Pulsed-Laser-Induced Dewetting: Probing the Assembly Dynamics of Nanoparticle Arrays
}

\author{
J.T. McKeown ${ }^{1}$, N.A. Roberts ${ }^{2}$, J.D. Fowlkes ${ }^{3}$, Y. Wu ${ }^{2}$, T. LaGrange ${ }^{1}$, B.W. Reed ${ }^{1}$, P.D. Rack ${ }^{2,3}$, and \\ G.H. Campbell ${ }^{1}$ \\ ${ }^{1}$ Condensed Matter and Materials Division, Lawrence Livermore National Laboratory, Livermore, CA, \\ USA \\ ${ }^{2}$ Materials Science and Engineering Department, University of Tennessee, Knoxville, TN, USA \\ ${ }^{3}$ Center for Nanophase Materials Science, Oak Ridge National Laboratory, Oak Ridge, TN, USA
}

The synthesis and organization of functional nanomaterials via bottom-up self- and directed assembly represents a critical challenge for the future of nanoscience. Generating arrays of nanoparticles with controlled size and spatial distributions is key to this challenge, and processes that exploit morphological instabilities, such as liquid-phase or solid-state dewetting, offer the potential to attain these fine-scale spatially correlated structures. Here, we present results from in-situ observations [1] revealing the assembly dynamics in metallic thin films using the high spatio-temporal resolution of the dynamic transmission electron microscope (DTEM) [2].

Pure Ni and 50-50 at.\% Cu-Co films were de magnetron sputter deposited directly onto 15-nm-thick silicon nitride TEM membranes. The films were $\sim 10 \mathrm{~nm}$ thick and polycrystalline with grain sizes on the order of the film thickness. Pump-probe in-situ experiments were conducted in the DTEM by laserheating the films using a 1064-nm wavelength, $~ 15-n s$ pulsed laser source with a Gaussian beam profile $\left(1 / \mathrm{e}^{2}\right.$ diameter of $\left.135 \pm 5 \mu \mathrm{m}\right)$ and imaging the specimen at a desired time delay after the heating pulse with an $\sim 15$-ns electron pulse ( $>2 \times 10^{9}$ electrons per single pulse) generated by a second laser that stimulates a photocathode [2].

Figure 1(a) presents time-resolved bright-field TEM images of a Ni film as it dewets upon heating. The time-delay sequence of images shows progressive hole formation at larger radii (the center of the laser pulse is toward the lower left corner of the images), leading to saturated nanoparticles. Figure 1(b) shows an SEM image of the resultant nanoparticle array. Correlated time and length scales suggest that the liquid-phase assembly of thin Ni films into nanoparticle arrays is induced by a spinodal instability. Complementary finite difference simulations agree well with time-resolved imaging and diffraction. $\mathrm{Cu}-$ Co thin films were investigated to explore the interactions between phase stability and morphological instability during dewetting of alloy thin films. Figure 2(a) shows a time-delay series of images as a $\mathrm{Cu}-$ Co film dewets upon heating. The timescales for dewetting are significantly longer than with pure $\mathrm{Ni}$ films, suggesting a solid-state mechanism plays a role in these experiments. Figures 2(b) and 2(c) show the resultant core-shell nanoparticles that were generated by the dewetting process. Results from both thin film systems will be presented and implications for directing nanoparticle assembly in pure metallic and alloy thin films will be discussed [3].

\section{References:}

[1] McKeown, J.T. et al., Langmuir 28 (2012) 17168.

[2] LaGrange, T.B. et al., Ultramicroscopy 108 (2008) 1441.

[3] Work at Lawrence Livermore National Laboratory was performed under the auspices of the U.S. Department of Energy, Office of Science, Office of Basic Energy Sciences, Division of Materials 
Sciences and Engineering under Contract DE-AC52-07NA27344. Work at the University of Tennessee and Oak Ridge National Laboratory was supported by the U.S. Department of Energy, Office of Basic Energy Sciences, Division of Materials Sciences and Engineering.
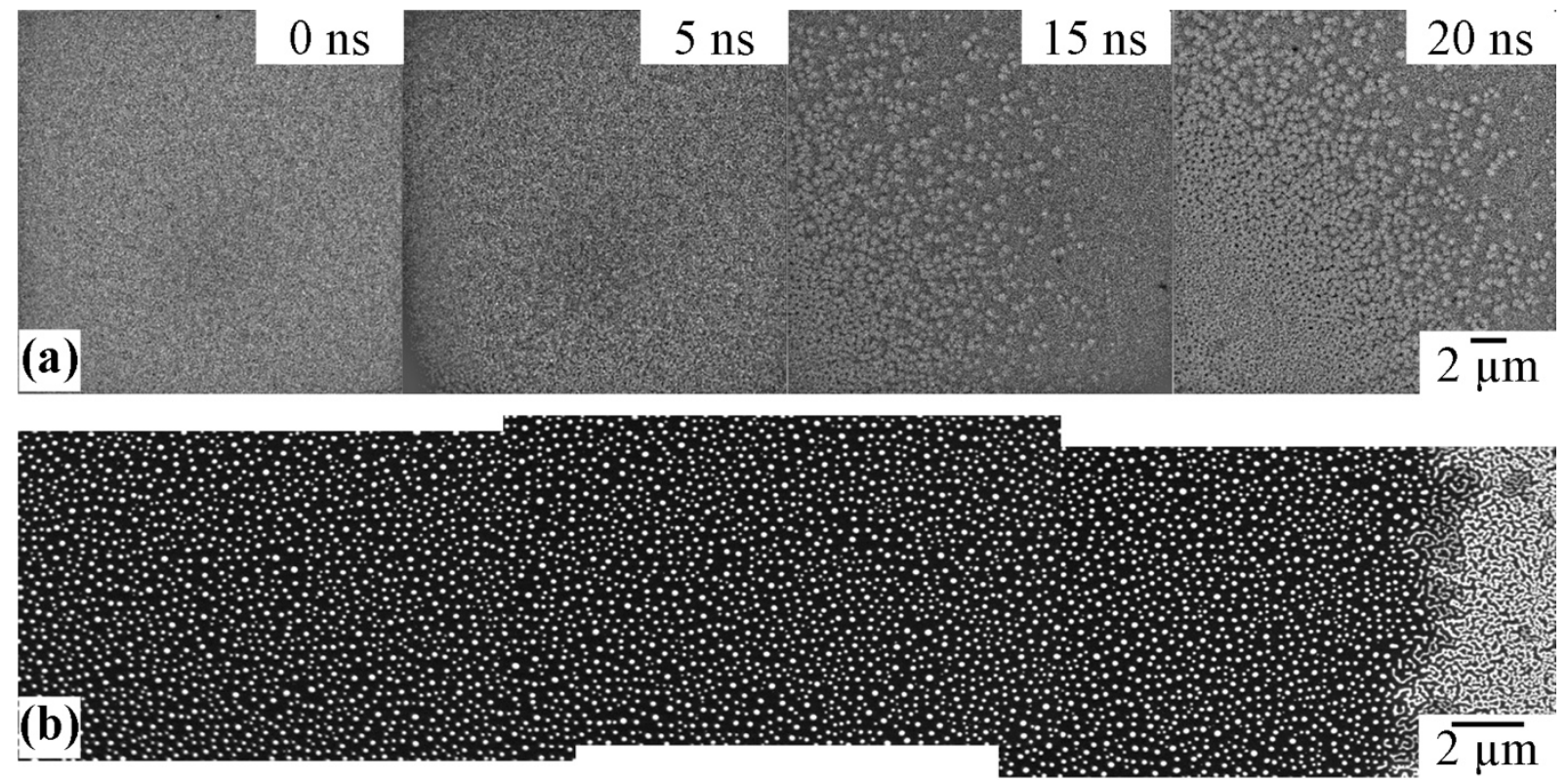

Figure 1. (a) Dynamic time-delay sequence of images of a 10-nm-thick Ni film as it dewets a silicon nitride membrane. The center of the laser used to initiate dewetting is toward the lower left corner of each image. (b) SEM image from center (left) to edge (right) across a laser-induced dewet region.

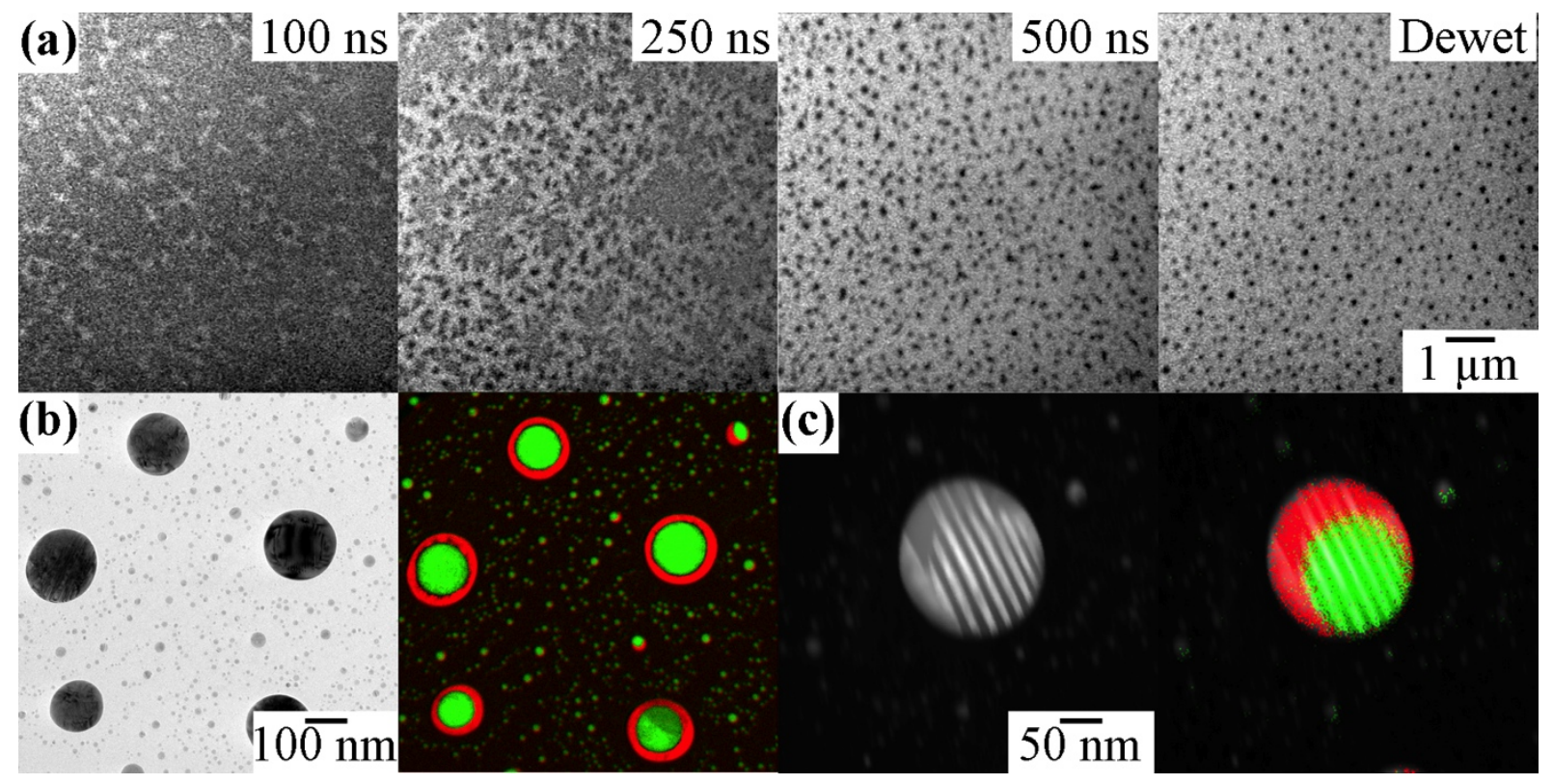

Figure 2. (a) Dynamic time-delay sequence of images of a 10-nm-thick $\mathrm{Cu}-\mathrm{Co}$ film as it dewets a silicon nitride membrane. (b) Zero-loss image and corresponding EFTEM composition map from a region of the dewet film. (c) Dark-field image of a twinned $\mathrm{Cu}-\mathrm{Co}$ nanoparticle and overlaid EDS composition map. In both composition maps, $\mathrm{Cu}$ is colored red and $\mathrm{Co}$ is colored green. 